International Conference on Renewable Energies and Power Quality (ICREPQ'11)

Las Palmas de Gran Canaria (Spain), 13th to 15th April, 2011

\title{
Synchronization control of parametric pendulums for wave energy extraction
}

\author{
A. Najdecka ${ }^{1}$, V. Vaziri, M. Wiercigroch \\ University of Aberdeen, Centre for Applied Dynamics Research, School of Engineering, \\ AB24 3UE, Aberdeen, UK, \\ e-mail: r02ajn9@abdn.ac.uk
}

\begin{abstract}
.
In this work the dynamics of a parametric pendulums system has been studied with a view to its application for sea wave's energy extraction. The idea is based on the conversion of the oscillatory motion of the waves into rotation of the pendulums. The system approximating a floating structure with two pendulums mounted on it has been modelled and analyzed. In the first stage of the study the general dynamics of the parametric pendulums has been investigated numerically and experimentally. The focus lies on synchronized rotational solutions, representing a most energetically favourable state of motion. The target state is to achieve a synchronized counter rotation of both pendulums. The controlling strategy, with the aim of initiating and maintaining the desired response, has been developed and verified numerically and experimentally. Different methods based on the delayed-feedback control have been suggested. The numerical and experimental results showing the difference in the system dynamics with and without control have been presented. Finally the energy extraction from the system has been simulated numerically and energy extraction control has been discussed.
\end{abstract}

\section{Key words}

Parametric pendulum, coupled pendulums, synchronization, delayed-feedback control, wave energy extraction

\section{Introduction}

The need to search for new alternative energy sources has been getting stronger in recent decades. The ocean waters despite being main solar energy collectors on earth, sill remain a highly unexplored energy source. Number of different technologies based on utilizing tides, oceans thermal energy and wave energy are being deployed however there is still need for further research in this area.

The motivation of this study is the idea of using the dynamics of the parametric pendulums for wave energy extraction. This concept has been proposed by Wiercigroch [1] and is based on the conversion of the kinetic energy of sea waves into rotational motion of the pendulum, mounted on the floating pontoon. The rotational motion of the single pendulum regarding this application has been studied in [2], [3]. The working principle of the parametric pendulum has been illustrated at the pictures below (Fig.1). The pivot point is subjected to harmonic excitation in vertical direction (Fig.1a). A parametric pendulum experiences different types of motion, which can be represented on the phase plane (Fig.1b). The region of oscillatory solutions (closed loops denoted by (1)) is bounded by a critical motion described by the separatrices (2). The response outside this region is denoted by (3) and corresponds to the rotation, which is of main interest for the energy extraction purposes. For stable rotations the solution on the phase plane needs to lie sufficiently far from the separatrix to ensure that the energy dissipation will not cause the pendulum to go back to the potential well inside the region bounded by separatrices. Once the pendulum rotates its energy can be extracted directly from the rotating shaft at the pivot point, which will be the scope of the following studies.

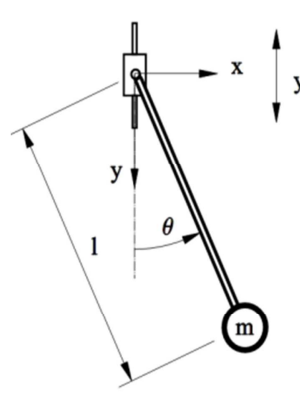

(a)

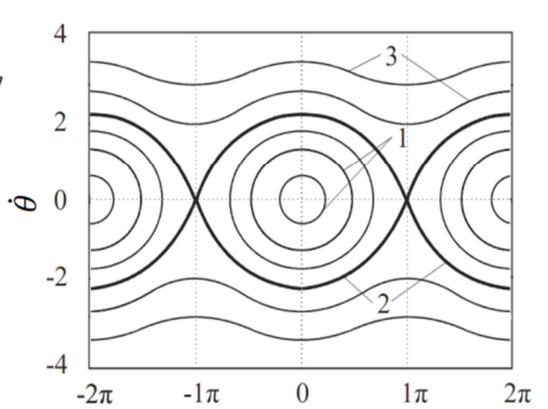

(b)
Fig.1 Working principle of parametrically-excited pendulum. Schematic representation (a) and phase plane showing different responses in terms of pendulum displacemnet and velocity (b).

A floating structure containing the pendulums system, would be subjected not only to the excitation originating from the sea waves but also reaction forces related to the rotating pendulum acting on the pontoon. The objective of this project is to design a structure which would utilize these interactions for increasing the stability of the pontoon and rotational motion, rather than suppressing them. It can be achieved by employing the set of 
pendulums instead of a single one. To compensate for the effect, which a single rotating mass exerts on the pontoon the system needs to be composed of multiple counter rotating pendulums. Due to the stabilizing effect the synchronization of the rotating pendulums is considered to be the target state for the energy extraction. In this initial study the system consisting of two pendulums will be considered. This will provide an insight into dynamical behaviour of more complex system.

\section{Physical and mathematical model}

The first step towards understanding the dynamics of the two pendulums system includes building physical and mathematical model. The schematic representation of the system considered is shown in the Figure $2 \mathrm{a}$. It consists of two pendulums mounted on the commonly excited flexible supporting structure. In the fist stage of the study the system has been treated on the plane. It has been modelled as a four-degrees of freedom system, where $\mathrm{x}$ and $\mathrm{y}$ denote the displacement of the structure in horizontal and vertical direction, $\theta_{1}$ and $\theta_{2}$ describe the angular displacement form the downward zero position. A synchronized state can be achieved due to coupling effect of the elastic base, capable of transmitting vibrations between the pendulums. The mass of the pendulums is concentrated mainly on the bob and therfore treated as a point mass. The damping effect of the base and on the shaft of the pendulum has been modeled as a viscous damping. The harmonic excitation on the base has been assumed, giving a good approximation of the wave's motion. The non-dimensional equations of motion for the two rotational degrees of freedom are given by:

$$
\begin{aligned}
& \theta_{1}^{\prime \prime}+x^{\prime \prime} \cos \theta_{1}+\left(1+y^{\prime \prime}\right) \sin \theta_{1}+\gamma_{\theta} \theta_{1}^{\prime}-u_{1}+e_{1}=0, \\
& \theta_{2}^{\prime \prime}+x^{\prime \prime} \cos \theta_{2}+\left(1+y^{\prime \prime}\right) \sin \theta_{2}+\gamma_{\theta} \theta_{2}^{\prime}-u_{2}+e_{2}=0,
\end{aligned}
$$

where $u_{i}$ and $e_{i}(i=1,2)$ represent control and resistive torque terms respectively. The parametric excitation terms $x$ ", $y$ "included in the above equations are described by the equation of motion of the flexible supporting structure:

$$
\begin{gathered}
x^{\prime \prime}+\gamma_{x} x^{\prime}+\alpha_{x} x+ \\
+a\left(\theta_{1}^{\prime \prime} \cos \theta_{1}-\theta_{1}^{\prime 2} \sin \theta_{1}+\theta_{2}^{\prime \prime} \cos \theta_{2}-\theta_{2}^{\prime 2} \sin \theta_{2}\right)=0 \\
y^{\prime \prime}+\gamma_{y}\left(y^{\prime}+p_{y} \omega \sin (\omega \tau)\right)+\alpha_{y}\left(y-p_{y} \cos (\omega \tau)\right)+ \\
+a\left(\theta_{1}^{\prime \prime} \sin \theta_{1}+\theta_{1}^{\prime 2} \cos \theta_{1}+\theta_{2}^{\prime \prime} \sin \theta_{2}+\theta_{2}^{\prime 2} \cos \theta_{2}\right)=0
\end{gathered}
$$

where all of the system parameters are nondimesional. $\gamma_{\mathbf{x}}$ and $\gamma_{\mathbf{y}}$ are the damping coefficients of the base in horizontal and vertical direction respectively, $\alpha_{\mathbf{x}}$ and $\alpha_{\mathbf{y}}$ are the stiffness coefficients, $p_{y}$ is the forcing amplitude, $\omega$ is the forcing frequency, $\tau$ is the time, a is a mass ratio. All of the system parameters and variables have been rescaled with respect to the natural frequency of the pendulums. The nondimensional form of the system equations allows comparison between different models. The result form the small scale experiment can easily be transferred on the real size installation. By changing the length of the pendulum and its natural frequency in this way, the dynamic response of the system can be adjusted to the different forcing conditions (different wavelengths). The height or frequency of the sea waves cannot be adjusted. However once the optimal forcing parameters range for the nondimensional system is determined, the system parameters can be adjusted to preserve the desired dynamical properties for the given sea waves conditions.

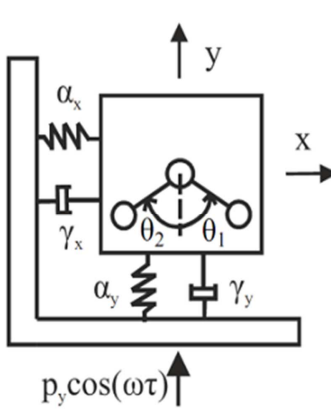

(a)

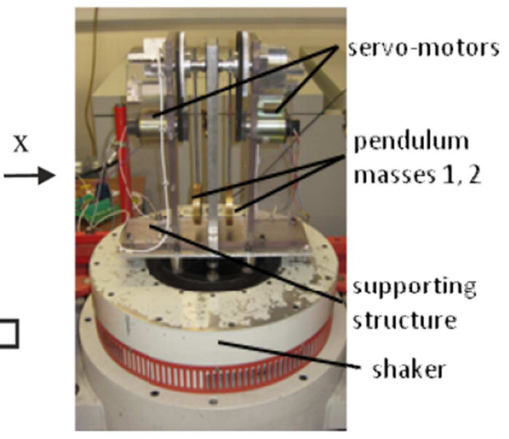

(b)
Fig. 2. Physical model of the two parametric pendulums system (a) and the corresponding experimental set-up (b).

\section{Numerical and experimental results}

The dynamics of the coupled pendulums system under harmonic excitation has been studied numerically and experimentally for different levels of forcing. First the system without any control algorithm has been examined. Secondly the controlling algorithm for maintaining synchronized rotation has been applied. The experimental studies have been carried out in the Dynamics Laboratory at the University of Aberdeen. The harmonic excitation of the system has been provided by the electromagnetic shaker. In the first stage of the study excitation acting only in the vertical plane has been considered (Fig.2a). The two pendulums have been fixed on the shaker as shown in the figure $2 b$ and the response of the system has been observed for different initial conditions and varying frequency and amplitude.

\section{A. System dynamics without control}

Without control the pendulums can experience several types of dynamic behaviour. For constant amplitude and frequency of forcing different synchronized solutions have been found including equilibrium points, oscillations, rotations, and rotations of one pendulum synchronized in phase with the oscillations of the second one (Fig. 3). Similar results have been obtained in the experimental studies (Fig. 4). Rotational solutions have been found in a wide range of forcing parameters (Fig.5). Additionally a natural tendency of the system to synchronize has been observed. Once the pendulums rotate the phase difference between them diminishes as the transient time elapses approaching one of the two synchronized solutions: in phase or in antiphase, depending on the initial conditions. However very specific initial conditions are necessary to initiate the rotational motion (Fig.7). Consequently a robust control 
algorithm is necessary to both start up the rotational motion and then to maintain it.

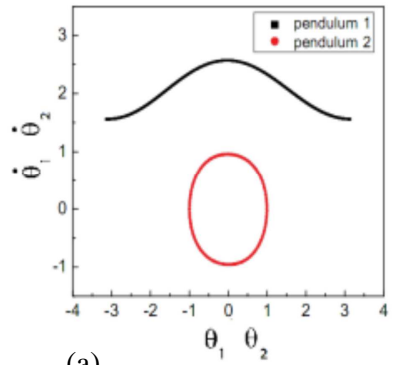

(a)

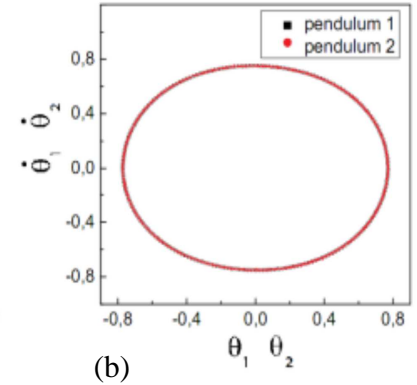

(b) pendulums. Once the motion is stabilized it can act as a generator extracting energy form the rotating shaft.
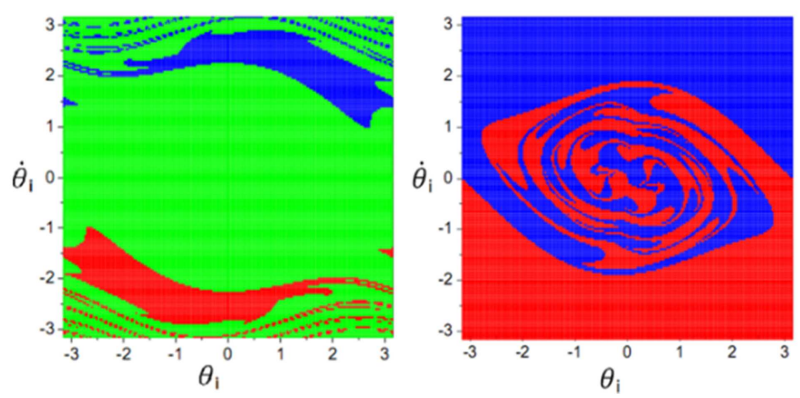

Fig. 6. Basins of attraction of the pendulum without (a) and with (b) control, where green marks the points corresponding to the initial conditions resulting in oscillations, blue in clockwise and red in anticlockwise rotation.

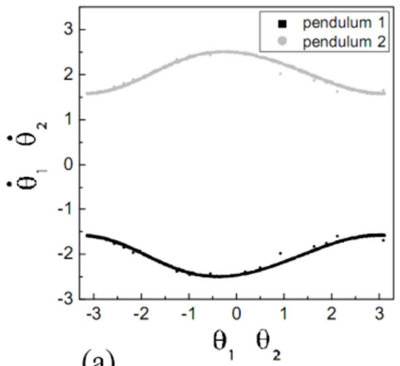

(a)

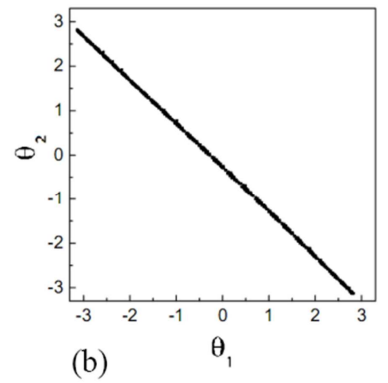

(b)
Fig. 4 Experimentally observed synchronized counter rotation of the pendulums for $\omega=2, p=0,07$. Phase plane showing the velocity of the pendulum against its displacement for each revolution (a) and displacement of the pendulum 2 as a function of pendulum 1 displacement (b), perfect correlation indicates complete synchornization.

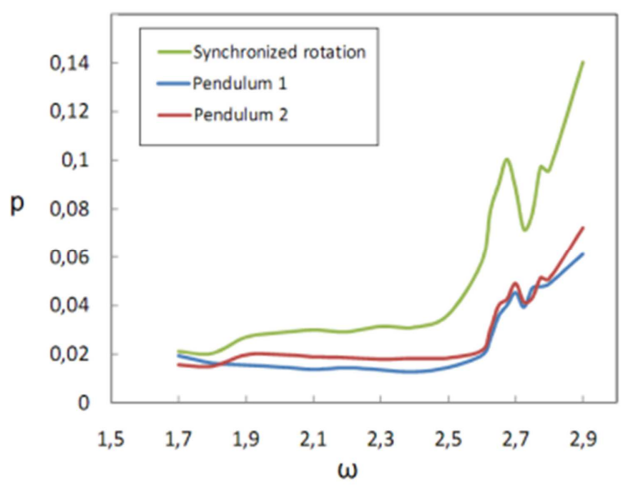

Fig. 5 Experimentally determined lower boundary of rotational motion in the excitation parameter space. The sudden jump has been observed around $\omega=2.7$, which corresponds to the natural frequency of the body of the shaker and is possibly the result of stronger pendulum-shaker interactions in this parameter region.

\section{B. Response with the control}

A target state for wave energy extraction requires rotational motion to be maintained irrespective of the changes in excitation. The control algorithm needs to be applied to both pendulums to ensure they synchronized rotational response. In the experimental study the contr( (a) g action is performe( (b) $/$ two servo-motor encoders attached to the penduiums shafts, working alternating as a motor, to provide the torque necessary to initiate rotational motion or to synchronize the two
After investigating several control techniques for parametric pendulum the delayed- feedback method has proved to be most efficient and robust. In this method the system can be stabilized by a feedback perturbation proportional to the difference between the present and a delayed state of the system [6], like the difference between velocities or angular displacements. During this study it has been observed that when applying two independent rotation controllers to two pendulums, their responses would naturally synchronize. Therefore the separate controlling signals supplied to pendulum one and two are given by:

$$
u_{i}=k \operatorname{sgn}\left(\dot{\theta}_{l}(t)\right)\left(\theta_{i}(t-\tau)-\theta_{i}(t)+2 \pi\right)
$$

where $\mathrm{k}$ is the proportionality constant, $\dot{\theta}_{\mathrm{i}}(\mathrm{t}), \theta_{i}(\mathrm{t})$ and $\theta_{i}(t-\tau)$ are the current velocity, current and delayed angular position of the i-th pendulum. The delay time $(\tau)$ is equal to the period of the desired periodic orbit, which for the period one rotations, being the dominant rotational response for the system considered, it can be determined from the period of the excitation. This can be extracted form the readings of the accelerometer measuring the oscillations of the pendulum base. If the pednlum rotates exactly with the period of excitation the angular displacement will increase $2 \pi$ for each revolution and at the same time the control variable $u_{i}$ will go to zero. The experimental and numerical result of applying two separate control signals, given by eqation (3) to initate and mainataine rotational motion are shown in the figures 9 and 10 .

Another approach to synchronizing and controlling the system is to make use of the interactions between the two pendulums and use the information about the state of one of them as a control input for the second one. The idea is to apply the delayed-feedback control to maintain the rotation of one of the pendulums and synchronize the second one with the first one. The velocity difference between the driving and driven pendulum has been used as a second control signal. By this control algorithm the pendulums are coupled according to the master-slave scheme, where the 'slave' pendulum is forced to synchronize with the 'master'. 


$$
u_{s}=k_{s}\left(\dot{\theta}_{1}(t)+\dot{\theta}_{2}(t)\right)
$$

where $\boldsymbol{u}_{\boldsymbol{s}}$ is the synchronization control variable, $\boldsymbol{k}_{\boldsymbol{s}}$ is the proportionality constant. The control variable will become zero when the instantaneous velocities of the pendulums will be exactly opposite. The control signal described by equation (3) is supplied to pendulum one, playing a role of the 'master' and while the control signal (4) is driving the second 'slave' pendulum. The numerical results of application of this type of controller are shown at the figure 8 .

(a)

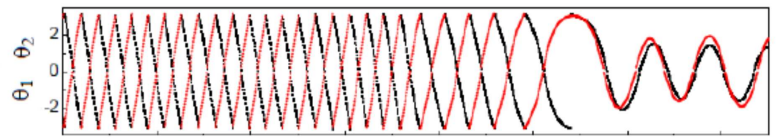

(b)

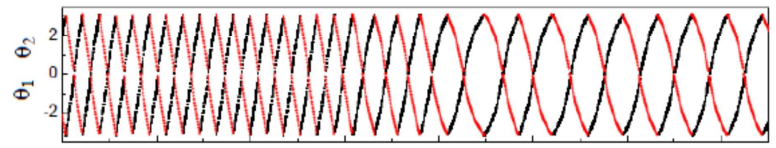

(c)

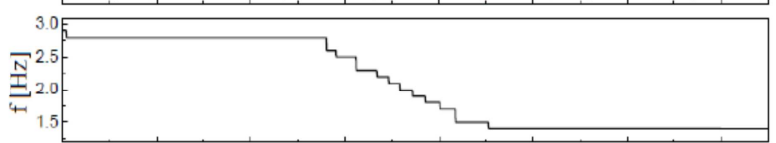

(d)
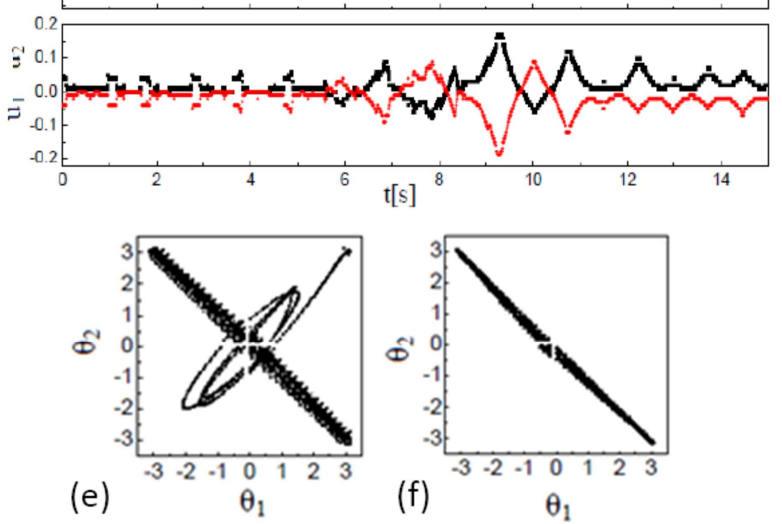

Fig.7 Experimental results showing the difference in the response of the system with and without control algorithm, while the forcing frequency is being changed from $3 \mathrm{~Hz}$ down to $1 \mathrm{~Hz}$. (a) displacements with no control, (b) displacements with control, (c) forcing frequency, (d) control signals, correlation between the displacements of the two pendulums without (e) and with control(f).

The two functions of the control include initiating the rotational motion irrespective of the initial conditions and in case of sudden changes in the excitation ensuring that the motion is sustained and synchronized. Fig. 7 shows the numerical result of the control application on the basins of attractions structure, the rotational motion can be initiated form every point on the phase plane. Fig. 8 shows the experimental results of applying a control algorithm to maintain the synchronized rotation, while the excitation frequency is being varied. The synchronized rotation has been successfully maintained.

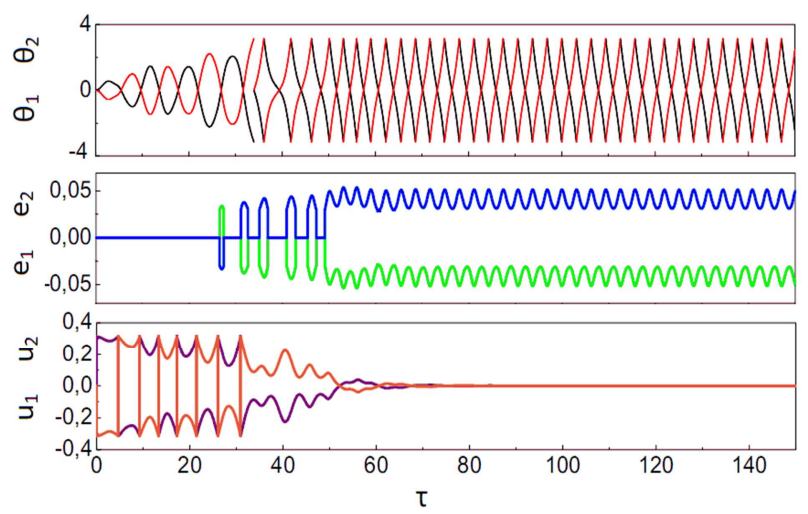

Fig. 8 Numerical time histories showing the displacement, resistive torques corresponding to the energy extraction and controlling terms versus time, while initiating and maintaining synchronized rotational motion of two pendulums with two identical controllers.

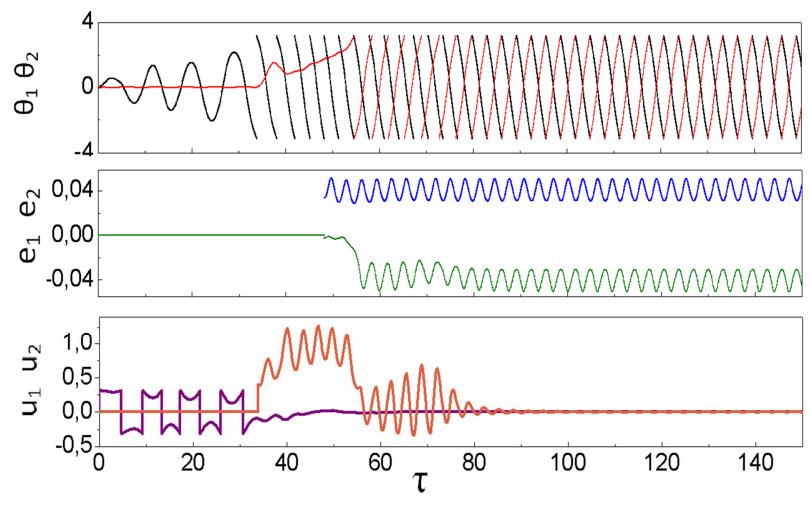

Fig. 9 Numerical time histories showing the displacement, resistive torques corresponding to the energy extraction and controlling terms versus time, when using the second synchronization control method.

\section{Energy extraction}

To maximize the amount of energy which can be extracted in each forcing cycle, without destroying rotational motion the energy extraction function proportional to the velocity of the pendulum has been proposed. The resistive torque applied to each pendulum shaft is given by:

$$
e_{i}=E_{i} \dot{\theta}_{i}(t)
$$

where $E_{i}$ is the energy extraction coefficient. Determination of the optimal value of $E_{i}$ is a crucial task. The maximum $E_{i}$ which does not destroy rotational motion depends on the forcing parameters $(\omega, \mathrm{p})$. Figure 11 shows a determined numerically surface of maximum energy extraction coefficient depending on the forcing parameters. 


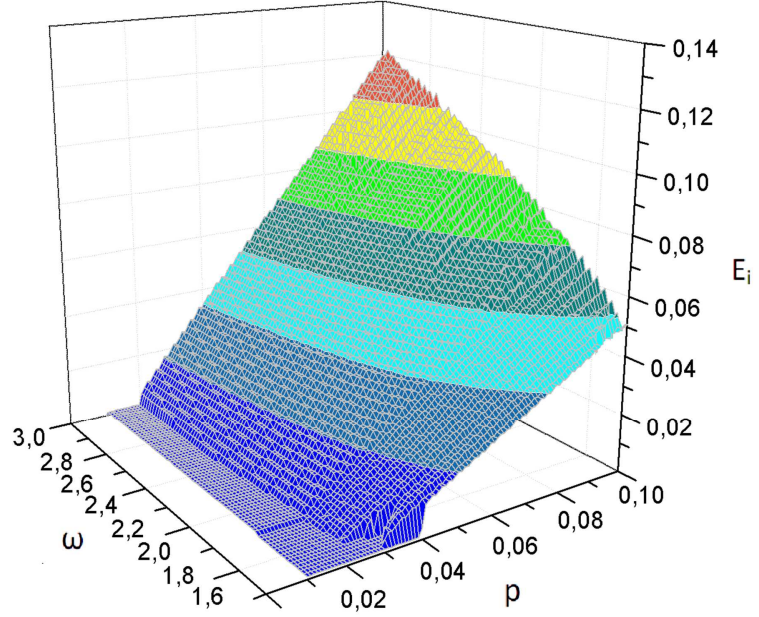

Fig. 10 Numerically determined $\mathrm{E}_{\mathrm{i}, \max }$ depending on the excitation parameters.

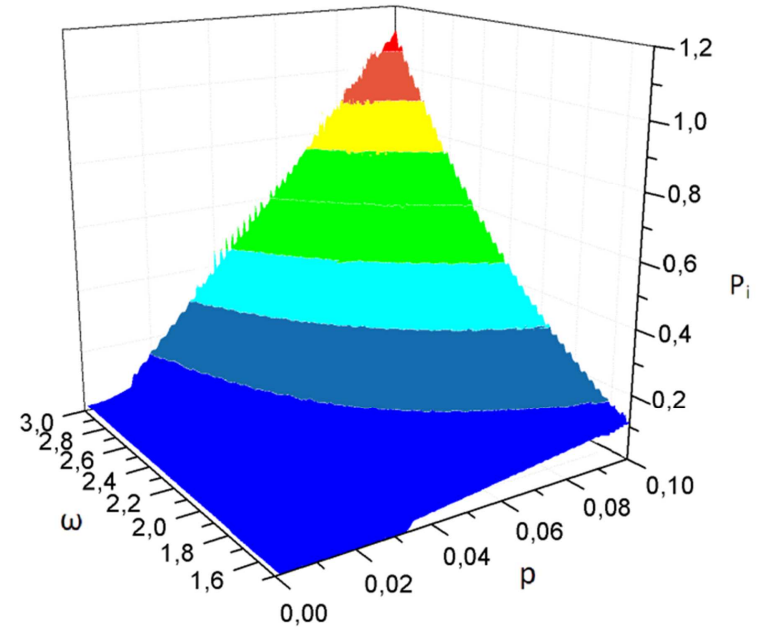

Fig. 11 Numerically determined maximum extracted power $P_{i}$ depending on the excitation parameters.

The power which can be extracted is a product of the resistive torque and pendulum velocity and expressed in nondimensional coordinates is equal to:

$$
\boldsymbol{P}_{i}=\boldsymbol{e}_{i} \dot{\theta}_{i}(t)=\boldsymbol{E}_{i} \dot{\theta}_{i}(t)^{2}
$$

$P_{i}$ strongly depends on the velocity of the pendulum. Therefore using rotational motion, characterised by significantly higher velocities than in oscillatory mode (Figure 3a) is more efficient than using the energy of oscillations. Additionally, it has been found in the experimental studies, that the friction related energy losses on the pendulum shaft are much higher for the oscillations around zero position than for the continues rotational motion [4].

The amount of energy which can be extracted needs to be controlled, using the information about the excitation, extracted from the base motion and the response of the pendulum. The variable $\mathrm{u}_{\mathrm{i}}$ can be used as a control input, so that the energy extraction occurs when the motion is already stabilized. So that the resistive torque applied to the shaft is given by:

$$
e_{i}=E_{i}(p, \omega) \dot{\theta}_{i}(t) s_{i}
$$

Where

$$
s_{i}= \begin{cases}1 & \text { for } u_{i} \cong 0 \\ 0 & \text { for } u_{i} \neq 0\end{cases}
$$

\section{Closing remarks}

In this study it has been found that the synchronized rotation of a two pendulums system is a stable motion in a wide range of forcing parameters. Moreover this motion can be successfully controlled with a low energy input. Alternative control techniques have been presented and assessing their efficiency for more complex forcing conditions will be the scope of further study. It has been shown that the synchronized rotational motion is still preserved when applying additional resistive torque on the shaft of the pendulum. The aim of the further research is to develop an energy extraction controller, which would allow extracting the energy surplus form the rotating pendulums while still maintaining their rotation. To maximize the energy output over one rotation the extraction term should vary periodically and be proportional to the angular velocity of the pendulum. The behaviour of the system with the proportional energy extraction term included has been simulated numerically and will be the studied within further experimental studies. The following step of the experiments includes moving studying the behaviour of the system in a wave tank.

\section{References}

[1] Wiercigroch M. Wave Energy Extraction via Parametric Pendulum (private communication), 2005.

[2] X. Xu, M. Wiercigroch, and M. P. Cartmell. Rotating orbits of a parametrically excited pendulum. Chaos, Solitons and Fractals, 2005 23:1537-1548.

[3] $\mathrm{X}$. Xu and Wiercigroch M. Approximate analytical solutions for oscillatory and rotational motion of a parametric pendulum. Nonlinear Dynamics, 47:311-320, 2007.

[4] Horton B., Wiercigroch M., and Xu X. Transient tumbling chaos and damping identification for parametric pendulum. Phil. Trans. R. Soc. A, 366:767-784, 2008.

[5] Pikovsky A., Rosenblum M., and Kurths J. Synchronization. A universal concept in nonlinear sciences. Cambridge University Press, 2001.

[6] Pyragas K. Continuous Control Of Chaos by SelfControlling Feedback. Physics Letters A. 1992, 421:428. 1992. 